\title{
Complex Network Analysis and Nonlinear Dynamics
}

\author{
Luis M. Varela and Giulia Rotundo
}

\begin{abstract}
This chapter aims at reviewing complex network and nonlinear dynamical models and methods that were either developed for or applied to socioeconomic issues, and pertinent to the theme of New Economic Geography. After an introduction to the foundations of the field of complex networks, the present summary introduces some applications of complex networks to economics, finance, epidemic spreading of innovations, and regional trade and developments. The chapter also reviews results involving applications of complex networks to other relevant socioeconomic issues.
\end{abstract}

Keywords Complex networks - Computer simulations - Market and financial models - Regional trade and development - Social networks - Statistical mechanics

\section{Introduction}

Complex networks have been proved during the latest decades as an essential formalism in order to describe the many situations in which agent-based models are required (Albert and Barabási 2002; Newman 2003; Pastor-Satorras et al. 2003; Boccaletti et al. 2006; Newman et al. 2006). Starting with the papers of Watts and Strogatz (1998) and of Barabási and Albert (1999) in the late nineties, this formalism has been accepted in the basic tool set of a growing number of disciplines like physics, biology, computer science, sociology, epidemiology, and economics among

\footnotetext{
L.M. Varela ( $\square)$

Grupo de Nanomateriais, Fotónica e Materia Branda, Departamento de Física da Materia

Condensada, Universidade de Santiago de Compostela, Campus Vida s/n. E-15782, Santiago de

Compostela, Spain

e-mail: luismiguel.varela@usc.es

G. Rotundo

Department of Methods and Models for Economics, Territory and Finance, La Sapienza

University of Rome, via del Castro Laurenziano 9, I-00161 Rome, Italy

e-mail: giulia.rotundo@gmail.com
}

P. Commendatore et al. (eds.), Complex Networks and Dynamics,

Lecture Notes in Economics and Mathematical Systems 683,

DOI 10.1007/978-3-319-40803-3_1 
others. This is so, because the skeleton of any complex system can be described by a network arising from the interactions of the many parts of which it is made up of. Hence, network theory is now considered to be one of the pillars in the theory of complex systems.

Complex system theory investigates how relationships between parts give rise to the collective (emergent) behaviors of a system and how the system interacts and forms relationships with its environment. The definition of complex systems is not unanimous, but one of the most popular visions states that a complex system is one made up of many parts with nonlinear interaction between them. These systems are open, nested systems, with memory and feedback loops, which may show emergent patterns and are structurally founded in dynamic networks of multiplicity. This definition emphasizes the importance of both the network of the system constituents and the nonlinear coupling between them. Therefore, one should expect that the relevant dynamics of complex systems is inherently nonlinear, and so that a combined study of complex networks and dynamic systems is a need for the development of the field of complex systems. Undoubtedly, structure decisively conditions the behavior of a dynamic system, and hence the interactions among the massive amount of their individual parts must be collectively considered, which is the main role of the network analysis. Indeed, the relationship between the dynamics and the topology of complex networks is a nowadays a central issue of network theory and, therefore, in its applications (see, e.g. Wang 2002 and Boccaletti et al. 2006 and references therein). Networks of coupled dynamical systems have been extensively considered in nonlinear dynamics since they can exhibit non trivial emergent phenomena, such as autowaves, Turing patterns, spiral waves, and spatiotemporal chaos, with applications to a plethora of large-scale real systems (Chua 1998). Specifically, synchronization phenomena have been extensively considered, as well as the evolution of the networks themselves (rewiring, growth, and so on) (Boccaletti et al. 2006).

In the present chapter, we briefly review the fundamental structural and dynamic properties of complex networks, and we consider some applications of this formalism to the understanding of nonlinear phenomena, including epidemiological models, synchronization in networks and financial contagions, games on networks, and clustering of network nodes and geographical information. Moreover, we present some novel results for epidemiological models of flu and sexually transmitted diseases (STDs), and we review the mean-field theory of contagion in networks.

The chapter is divided in four sections. The first one is this introduction, the second contains a review of the theory of complex networks; in Sect. 3 we present some applications of this theory to the analysis of nonlinear phenomena, including some novel results for flu and STDs and, finally, Sect. 4 summarizes the main conclusions of the chapter. 


\section{Theory of Complex Networks}

In this section we briefly review some of the main results in the theory of complex networks developed during the last two decades. The theory of complex networks has evolved from the branch of mathematics known as graph theory, within which these objects were traditionally considered. Probably the best known of all of them-besides the regular graph or lattice-was Erdös and Renyi's random graph (Erdös and Rényi 1959).

Probably the most significant result in the contemporary theory of networks was the introduction of small-world networks by Watts and Strogatz (1998) (WS), trying to describe the transition from a regular lattice to a random graph. Beyond its mathematical interest, this new type of network successfully gives account of the "six degrees of separation" concept, uncovered by the social psychologist Milgram in the late 1960s (Milgram 1967). This is a well-known emergent property of many social networks in which the average path length (i.e. the average number of steps one has to take across the network for connecting two given individuals) is notably smaller than that in a regular lattice. Purely random networks also exhibit the smallworld property, but, as we shall see, they give rise to far sparsely connected networks (low clusterization) to accurately describe social networks.

The second major advance in the theory of complex networks took place the year after Watts and Strogatz's paper in Nature, when Barabási and Albert (1999) introduced scale-free networks, which mimic many situations where the networks show vertex connectivities that follow a scale-free power-law distribution. They were able to prove that this emergent property followed from the continuous expansion of the network due to the addition of new vertices, and the preferential attachment of the new nodes to those sites that are already well connected. Of course, this new type of network showed the small-world property, but, in contrast with WS networks, they were able to describe situations where a high degree of heterogeneity between the agents' connectivity exists. This new type of network completed the network ecosystem, and the theory was then in place to describe any possible connection pattern in agent-based systems, which opened the door to the explosive expansion of the theory of complex networks themselves and their applications. In what follows we summarize some of the most important results reported since then for the structure and dynamics of these fascinating objects.

A network (graph) is formally described as a pair $(V, E)$, where $V$ is a set of nodes (vertices), and $E$ is a set of links (edges) defined by two nodes that represent the source and the end of the link Suppress. The assignment of labels to the elements of the network is called graph labelling, and the special case of coloring is among the most important labelling schemes. A graph is said to be $k$-colorable if it can be assigned a $k$-colouring, i.e. a labelling of the graph's vertices with colors such that no two vertices sharing an edge have the same color. The smallest number of colors needed to color a graph $G$ is called its chromatic number, and is often denoted as $\chi(G)$. Associated to this concept is that of chromatic polynomial, which counts the 
number of ways a graph can be coloured using no more than a given number of colors.

Networks can be classified according to several criteria:

1. According to the directionality of the links, they can be either directed or undirected graphs.

2. Networks can be classified as weighted or unweighted depending on whether different weights are associated to their edges.

3. Sparse and fully connected networks differ in the fraction of interconnected nodes.

4. Depending on their time evolution, networks can be classified into static and evolving.

5. Depending on their node degree probability distribution, they can be classified as: regular (deterministic), exponentially distributed Watts-Strogatz networks, scale-free Albert-Barabási network, fully random graphs, general uncorrelated networks and others.

Another important type of networks are bipartite networks, formed by two disjoint sets of nodes $U$ and $V$ such that every node in $U$ is connected to a node in $V$. The adjacency matrix of a bipartite network is of the form:

$$
A_{i j}=\left(\begin{array}{ll}
0 & B \\
B & 0
\end{array}\right)
$$

Some important properties of these networks are:

(a) A graph is bipartite if and only if it does not contain an odd cycle.

(b) A graph is bipartite if and only if its chromatic number is less than or equal to 2 .

(c) The spectrum of a graph is symmetric if and only if it's a bipartite graph.

The most frequently employed method for the representation of the vertex connectivity in networks with $N$ nodes is the adjacency matrix $A=\left(a_{i j}\right) \in \mathbb{R}^{N \times N}$, whose rows and columns are the nodes of the network, and whose term $a_{i j}>0$ corresponds to the weight of the link from node $i$ to node $j$, which in an unweighted network is always set to $a_{i j}=1$ for the non null elements (if the network is directional, other criteria have been introduced). In this case, and when the network is undirected -the links connecting two edges acts bidirectionally- the matrix $A$ is symmetric $a_{j i}=a_{j i}$ for all nodes $i, j$. The absence of links is given by zero elements $a_{i j}=0$. This matrix contains much useful information about the network including the so called spectrum of the graph, given by the set of eigenvalues of $A_{i j}$. On the other hand, a path from node $i_{h_{1}}$ to node $i_{h_{k}}$ in the network is a sequence of unique nodes $i_{h_{1}}, i_{h_{2}}, \cdots, i_{h_{k}}$ such that $a_{i_{h_{j}}, i_{h_{j}+1}} \neq 0$, and can be detected through the power of the adjacency matrix $A^{h_{k}}$. These paths are relevant for studying diffusion processes as well as the relevance of nodes. 
This matrix allows the calculation of others related to it such as the degree matrix,

$$
D_{i, j}:= \begin{cases}\operatorname{deg}\left(v_{i}\right) & \text { if } i=j \\ 0 & \text { otherwise }\end{cases}
$$

or the Laplacian matrix, $L_{i j}=D_{i j}-A_{i j}$ or

$$
L_{i, j}:= \begin{cases}\operatorname{deg}\left(v_{i}\right) & \text { if } i=j \\ -1 & \text { if } i \neq j \text { and } v_{i} \text { is adjacent to } v_{j} \\ 0 & \text { otherwise }\end{cases}
$$

Spectral graph theory is the study of the properties of a graph by means of the characteristic polynomial, eigenvalues, and eigenvectors of its adjacency matrix or Laplacian matrix. Some interesting results are: (1) zero is always an eigenvalue of this matriz since the sum of all the elements of every row and column is zero. Indeed, the multiplicity of this eigenvalue gives the number of connected components of the graph. (2) As usual, the smallest non-zero eigenvalue of the Laplacian matrix gives the spectral gap, and its second smallest eigenvalue is the algebraic connectivity (or Fiedler value) of the graph, which measures the connectivity degree of the graph, with applications in robustness and synchronizability of networks.

\subsection{Structure of Complex Networks}

The analysis of the structure of networks is made using a set of relevant parameters that allow the classification of these objects. In the rest of this subsection we introduce some of the most important and frequently used (Fig. 1).
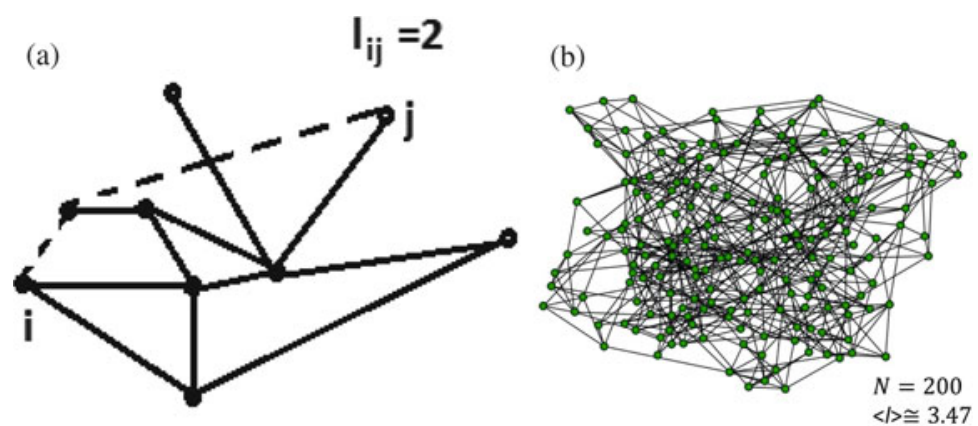

Fig. 1 (a) Example of chemical distance. (b) Average path length in a SW network with $N=200$ and $p=0.3$ and the number of connections to nearest neighbours in ring topology $k=6$ 
- Small-worlds: relatively short path between any two nodes, defined as the number of edges along the shortest path connecting them. The connectedness can also be measured by means of the diameter of the graph, d, defined as the maximum distance between any pair of its nodes. Networks do not support the definition of a "distance". They are no proper metric space although some attempts to introduce a metric on hidden metric space have been made Serrano et al. (2008). Hence, a chemical distance between two vertices $l_{i j}$ must be introduced, which is defined by the number of steps from one vertex to the other following the shortest path. Associated to this distance emerges the one of the most important measures for describing the structure of a complex network is the so called average path length between connected nodes

$$
<l>=\frac{2}{N(N-1)} \sum_{i<j} l_{i j}=\sum_{l} p(l)
$$

Contrary to what happens in a regular lattice -in which $\langle l>\sim \sqrt{N}$ - in most real networks, $<l>$ is a very small quantity (small-world) that scales with the number of nodes as $<l>\sim \log N$.

- Centrality Centrality measures the relative importance of the vertices of a network in terms of a real-valued function, where the values produced are expected to provide a ranking which identifies the most important node. Obviously, importance is highly dependent on the context and a criteria of importance must be provided. Several types of centrality have been introduced:

- Degree centrality: number of connections a node has, $C_{D}(v)=\operatorname{deg}(v)$. This is a direct measure of how exposed a given vertex $v$ is to whichever it flows through the network.

- Closeness centrality, defined for vertex $i$ as

$$
\tilde{C}_{i}=\frac{1}{\sum_{j} d_{i j}}
$$

where $d_{i j}$ is the distance of node $i$ to node $j$.

- Path centrality: To go from one vertex to another in the network, following the shortest path, a series of other vertices and edges are visited. The ones visited more frequently will be more central in the network.

- Betweenness centrality, number of shortest paths that pass through a given node for all the possible paths between two nodes. Measures the "importance" of a node in a network, and it is given by

$$
\begin{aligned}
C_{B}(v) & =\frac{\text { Number of shortest paths including } v}{\text { Total number of shortest paths }} \\
& =\sum_{s \neq v \neq t \in V} \frac{\sigma_{s t}(v)}{\sigma_{s t}}
\end{aligned}
$$


(a)

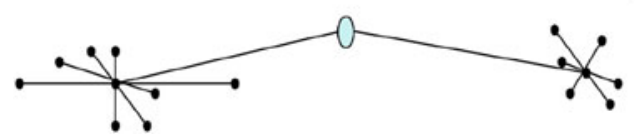

(b)

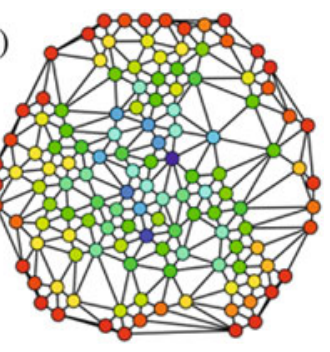

Fig. 2 (a) The node in blue has the largest betweenness centrality, as does the node in navy blue in (b) (Source of (b): Claudio Rocchini, Wikipedia)

- Eigencentrality: a measure of the influence of a node in a network (Fig. 2).

- Katz centrality: Katz centrality is a generalization of degree centrality that measures the number of all nodes that can be connected through a path, penalizing the contributions of distant nodes.

$$
C_{\text {Katz }}(i)=\sum_{k=1}^{\infty} \sum_{j=1}^{n} \alpha^{k}\left(A^{k}\right)_{j i}
$$

where $0<\alpha<1$ is an attenuation factor. This centrality measure is related to the very famous PageRank,

$$
P_{i}=\alpha \sum_{j} A_{j i} \frac{\tilde{C}_{j}}{L(j)}+\frac{1-\alpha}{N}
$$

with $L(i)=\sum_{j} a_{i j}$ is the number of neighbors of node $i$, used by Google to rank websites in their search engine results.

- Bonacich centrality, which allows for negative values of the attenuation factor $\beta$.

$$
C_{B O N}(i)=e_{i}^{T}\left(\frac{1}{\beta} \sum_{k=1}^{\infty}(\beta A)^{k}\right) \mathbf{1}=\frac{1}{\beta} \sum_{k=1}^{\infty} \sum_{j=1}^{n} \beta^{k}\left(A^{k}\right)_{i j}
$$

Closely related to those centrality coefficients is the so called core-periphery coefficient, introduced by Holme (2005). This core-periphery coefficient measure if the network is divided in a core that is both distance, and densely connected and central in terms of graph distance and a sparsely connected periphery, and it is defined as

$$
c_{c p}(G)=\frac{C\left[V_{\text {core }}(G)\right]}{C[V(G)]}-\left\langle\frac{C\left[V_{\text {core }}\left(G^{\prime}\right)\right]}{C\left[V\left(G^{\prime}\right)\right.}\right\rangle_{G \in \mathscr{G}(G)}
$$


where $\mathscr{G}(G)$ is the ensemble of graphs with the same set of degrees as G. and $V_{\text {core }}(G)$ is the $k$ core (i.e. a maximal subgraph with the minimum degree $k$ ) with maximal closeness.

- Clustering coefficient: This coefficient provides a quantitative measure of the degree to which links in a network follow a transitive property (i.e. if $i$ is linked to $j$, and $j$ is linked to $k$, then $i$ may or may not be connected to $k$ ). Such transitive links are very common in social networks, and realistic structures must take this into account. The local clustering coefficient of node $i$ of a set of $k_{i}$ nodes linked to node $i$ is defined as the ratio between the number $E_{i}$ of edges that actually exist between these $k_{i}$ nodes and the total number $C_{i}=k_{i}\left(k_{i}-1\right) / 2$. This clustering coefficient provides a measure of the local connectivity structure of the network, i.e. how close the local neighbors of a given node are to being a clique (complete graph). This coefficient was introduced by Watts and Strogatz (1998) to determine whether a graph is a small-world network. The global clustering coefficient is defined as the ratio between the total number of closed triplets and the total number of connected triplets of networks (Fig. 3). The average clustering coefficient

$$
<C>=\frac{1}{N} \sum_{j} C_{j}
$$

can be introduced, as well as the clustering spectrum for vertices of degree $k$,

$$
<C(k)>=\frac{1}{N p(k)} \sum_{j} \delta_{k_{j} k} C_{j}
$$

in the usual way.
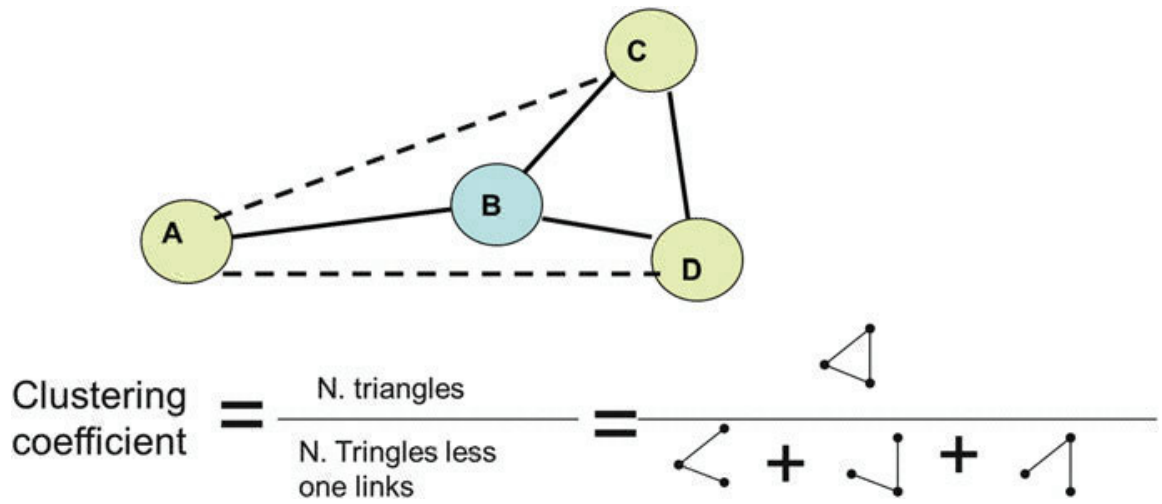

Fig. 3 Calculation of the global clustering coefficient 

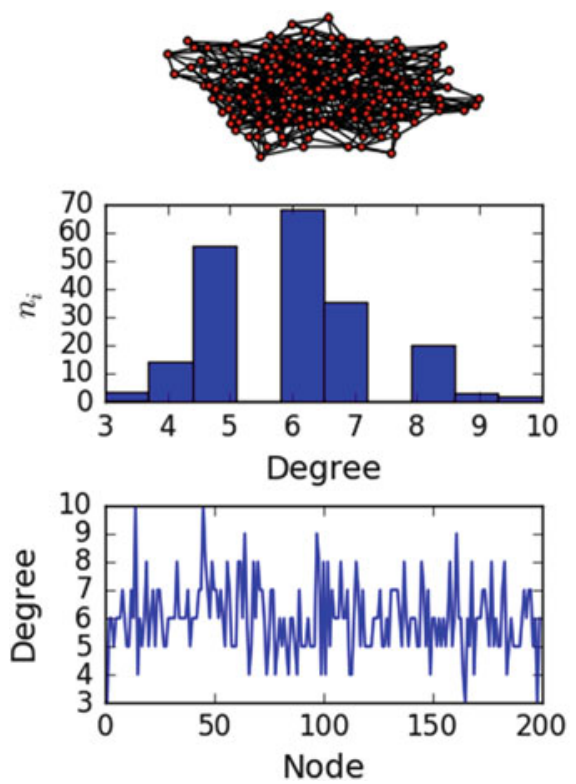
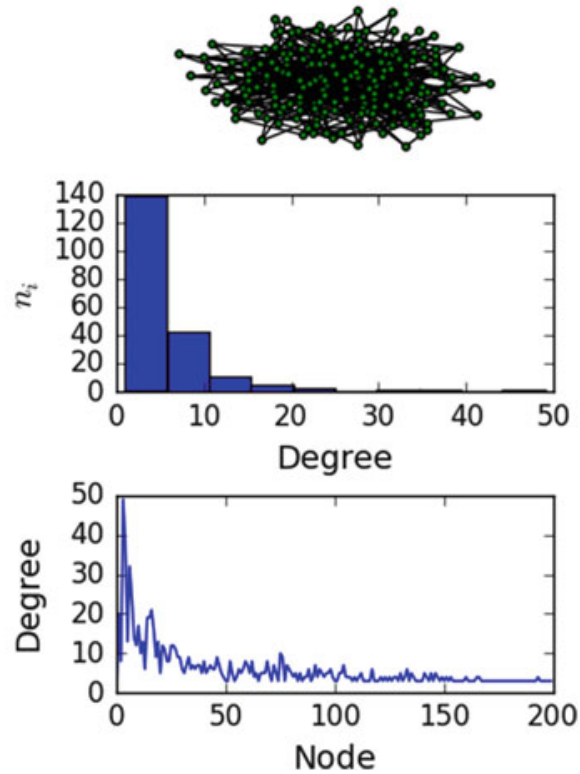

Fig. 4 WS distribution (left column) and AB distribution (right column)

- Degree distribution: The degree distribution, $p\left(k_{i}\right)$ is the probability that a given node $i$ has a definite number of edges, $k_{i}$. In directed networks an in-degree and an out-degree are to be defined, but in undirected network both coincide. These degree distribution can fall in two categories: those within the basin of attraction of the Gaussian distribution (exponential distribution with all their moments finite) and those in the basin of attraction of some other Lévy distribution (subexponential, fat-tailed distributions). The degree distributions associated to the main four types of complex networks introduced above are (Fig. 4 shows those for WS and AB networks):

$$
p(k) \sim \begin{cases}\delta\left(k-k_{0}\right) & \text { regular lattice } \\
\left(\begin{array}{l}
N \\
k
\end{array}\right) p^{k}(1-p)^{N-k} & \text { random network } \\
e^{-\gamma k} & \text { WS networks } \\
k^{-\alpha} & \text { AB networks }\end{cases}
$$

Of course, these distributions open the doors to defining the whole set of moments of the degree of nodes. Specifically, we can mention the average degree

$$
<k>=\frac{1}{N} \sum_{i} k_{i}=\sum_{k^{\prime}} k^{\prime} p\left(k^{\prime}\right)
$$

A network is called sparse if its average degree remains finite when taking the limit $N \gg$. In real (finite) networks, $<k>\ll N$. Of course, real networks are 
usually correlated, i.e. the degrees of the nodes are not in general independent. This must be described by a conditional probability distribution $p\left(k^{\prime} \mid k\right)$ which represents the probability of a $k$-node pointing to a $k$-node. For an uncorrelated network,

$$
p\left(k^{\prime} \mid k\right)=\frac{k^{\prime}}{<k>} p\left(k^{\prime}\right)
$$

independent of $k$, while for a correlated network this probability depends on the degree of both linked nodes. $p(k)$ and $p\left(k^{\prime} \mid k\right)$ are not independent, but they are related by a detailed balance condition arising form the fact that the number of nodes of degree $k$ linked to nodes of degree $k^{\prime}$ equals the number of nodes of degree $k^{\prime}$ linked to nodes of degree $k$. On the other hand, in terms of these conditional probability the average degree of the nearest neighbours of vertices of degree $k$ can be calculated as

$$
<k_{n n}>=\sum_{k^{\prime}} k^{\prime} p(k \mid k)
$$

If $<k_{n n}>$ is an increasing (decreasing) function of the degree $k$ the mixing is assortative (disassortative) meaning that highly connected nodes tend to be linked to other highly (lowly) connected nodes, i.e. there exists a bias in favor of connections between network nodes with similar (dissimilar) characteristics (Pastor-Satorras et al. 2003).

- Nestedness index (Araujo et al. 2010). This concept was originally introduced in the context of ecological studies (Atmar and Patterson 1993). A bipartite network formed by islands and species_-linked if the former inhabits the latter-is said to be nested if the species that exist on a few islands tend always to be found also on those islands inhabited by many different species. It indicates the likelihood of a node being linked to the neighbours of the nodes with larger degrees. The mean topological overlap between nodes (Almeida-Neto et al. 2008) has been introduced to quantify nestedness.

\section{Dynamics of and on Complex Networks}

We have previously mentioned that complex networks are at the root of the structure and dynamics of complex systems. As usual -and in this case probably to a much larger extent than in simple conventional systems- there exists a strong interplay between structure and dynamics, so it is difficult to say if the observed equilibrium structure is a cause or a consequence of the dynamics. Without entering into such philosophical questions, there are different dynamic processes that one can consider in complex networks:

- Synchronization of networks and collective dynamics. 
- Evolution of the network itself (evolving networks, rewiring).

- Spreading processes (epidemics, rumors).

Closely associated to dynamics is the concept of robustness, which can be defined as the ability of the network to withstand failures and perturbations. These concepts have been extensively reviewed in the past (see the reviews of Boccaletti et al. 2006 and of Arenas et al. and the references therein Arenas et al. 2008) and the reader is referred to this literature for in-depths treatments.

\section{Nonlinear Phenomena on Complex Networks}

This section shows some applications of nonlinear dynamics on complex networks for the study of economic and financial problems. The need for new and more fundamental understanding of economic and financial crises has fostered the development of studies on the structure and dynamics of economic and financial networks. In such networks, nodes may represent firms, banks, or even countries, and links represent their mutual interaction, be it cross-ownership, credit-debt, or trade relationships. The network structure allows to focus on the propagation of shocks and on resilience, which show quite different behavior depending on the structure of the network (da Fontoura Costa et al. 2011; Schweitzer et al. 2009a). This section is organized in four subsections: Epidemiological models, Synchronization in networks and financial contagions, Games on networks, and Clustering of network nodes and geographical information.

\subsection{Epidemiological Models}

Understanding risk, unfolding different sources of risk, preventing failure on large systems, spreading of crises, and large crashes, are among the major tasks to undertake in modern economic and financial literature. Conventional epidemiological models have been used as models of propagation and contagion well beyond the limits of medical epidemiology. They have indeed served as models of any kind of diffusion in social and economic contexts, where large populations with a complex pattern of interaction among agents exist (spreading of rumours or information, diffusion in markets and financial markets, etc.). From this perspective, epidemiological models have also been reconsidered in terms of transmission of financial shocks. For instance, the average lifetime and persistence of viral strains on the Internet is examined in Pastor-Satorras and Vespignani (2001). It so happens that the spreading pattern depends crucially on the properties of the social network and on the diffusion (or contagion) mechanism underlying the complex network (López-Pintado 2008; Barrat et al. 2008). A key factor is the scale-free feature of the network, and a relevant result is the absence of a critical threshold for the 
spread of computer viruses. That means that any virus is going to propagateunless properly blocked (Pastor-Satorras and Vespignani 2002). In Barthélemy et al. (2005) simulation studies proved that assortative networks have an early burst, against the late spread in disassortative networks. This also gives recommendations on vaccination policies: blocking the infection at the level of hubs is the optimal choice.

Compartmental models are a special kind of epidemic models that split the population over a discrete set of states (or compartments) depending on their health status with respect to the disease. Individuals can change state as time goes by. Probably the best known of all these models is that by Kermack and McKendrick (1927), a cornerstone in the mathematical modelling of epidemics introduced in order to understand the evolution of infected patients observed in epidemics such as the London (1665-1666) or Bombay (1906) plagues or cholera (London 1865). This deterministic model uses three compartments corresponding to Susceptible (S), Infected (I) and Recovered (R) for classifying the population, whose size is supposed to be fixed (i.e., no births, deaths due to disease, or deaths by natural causes). No incubation period is allowed so the disease is passed instantaneously and a final immunity is acquired by the individuals. The mechanism that the pathogen uses for its propagation is the direct contact between the susceptible and the infected individuals, assuming homogeneous mixing of a completely homogeneous population (no age, spatial, or social structure). This hypothesis states that the per capita rate of acquisition of the disease by the susceptible individuals is directly proportional to the density of infective individuals, and it is a typical mean-field assumption. These assumptions leads to the following set of equations (Kermack and McKendrick 1927):

$$
\begin{aligned}
\frac{d S(t)}{d t} & =-\beta S(t) I(t) \\
\frac{d I(t)}{d t} & =\beta S(t) I(t)-\gamma I(t) \\
\frac{d R(t)}{d t} & =\gamma I(t)
\end{aligned}
$$

These compartmental models focus on a specific set of characteristics, and divide network nodes in subsets (compartments) that are homogeneous with respect to the specific characteristic. For instance, in a vaccination program, the study of the possible propagation of the infection can be done in accord to the different age ranges. In such context, it is natural to draw network links among the compartments. The relevance of the structure of the network has also been shown on the resilience: assortative networks resist random attacks better than others, but not to targeted attacks (Albert et al. 2000; Chen and Cheng 2015).

Underlying the epidemic spreading there is always a complex network defined by the contacts of the individuals that support the contagion. The topology of this network drastically determines the propagation of the disease. Essentially, 
in homogeneous exponentially distributed networks the number of contacts of a given individual can be replaced under certain circumstances by its average value, neglecting fluctuations in the number of contacts (mean-field theory). This is not possible in epidemic diseases where fluctuations in the number of contacts of the individuals are important (e.g. sexually transmitted diseases), but is relatively accurate in diseases like flu. For diseases travelling in homogeneous networks the infection rate $\beta$-which controls the epidemic dynamics together with the recovery rate, $\gamma$-is given by the probability of infection per contact, $\lambda$ times the average number of contacts of a given characteristic individual, $\beta=\lambda<k>$. This is, of course, not possible in the case of heterogeneous scale-free networks, in which the average number of contacts is not a good representative of the distribution, or fluctuations are too important to define an "average individual".

One of the main predictions of the SIR model is the existence of an epidemiological threshold, i.e. a cut-off for the infection rate below which the epidemic disease is not capable of infecting a significant fraction of the population and dies out. In the specific case of the Kermack-McKendrick SIR model this is given by the basic reproductive number

$$
R_{0}=\frac{\beta S}{\gamma}
$$

This variable quantifies the number of secondary infections coming from a primary case in a completely susceptible population, and $R_{0}=1$ is the critical value that divides the region where the outbreak peters out $\left(R_{0}<1, \dot{I}<0\right)$ from that where the epidemic spreads $\left(R_{0}>1, \dot{I}>0\right)$. The specific value $R_{0}=1$ corresponds to an endemic behaviour, with a finite fraction of the population infected in the asymptotic limit, $t \rightarrow \infty$.

The description of flu epidemic spreading demands an epidemiological model of the different states the individual goes through during the infection period. Moreover, the network structure must be provided, and since in this case the effect of hubs is negligible, it may be reduced to a mere number, the average connectivity of the network, $\langle k\rangle$, which amounts to a mean-field level of description. Furthermore, a good description of the epidemiology of the disease must be used. In our case, in order to describe the propagation of an epidemic of avian flu in Galicia (Spain) we proposed a compartmental model based on the well-known SEIR model, including an incubation period of exposed individuals $E(t)$ and the possibility that a fraction of those exposed to the virus recover without being effectively infective at any point of the process (see Fig. 5a). Moreover, we allowed for the seed of a fraction of infective individual from outside the region at a rate $\delta$. This is a deterministic model since the factors intervening in the process are controllable and a deterministic differential equation system governs the whole process (Fig. 5b). However, the most natural way to describe an epidemic disease is by means of stochastic models, which describe the process of propagation of the infective agent in a probabilistic way. These models are used when random fluctuations or heterogeneities of the system are important, such as in small or isolated populations. They have several 
(a)

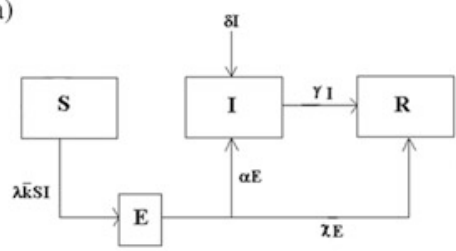

(b)

$$
\begin{aligned}
& \frac{d S(t)}{d t}=-\lambda \bar{k} S(t) I(t) \\
& \frac{d E(t)}{d t}=\lambda \bar{k} S(t) I(t)-\alpha E(t)-\chi E(t) \\
& \frac{d I(t)}{d t}=\alpha E(t)-\gamma I(t)+\delta I(t) \\
& \frac{d R(t)}{d t}=\chi E(t)+\gamma I(t)
\end{aligned}
$$

Fig. 5 Deterministic modified SEIR compartmental model (a) and its associated system of nonlinear differential equations (b)

advantages over the deterministic model, since they study the dynamic of the illness at an individual level instead of on a collective fashion. However, they normally demand numerical simulations. Deterministic models are approximations of the corresponding stochastic models (Nåsell 2002) where the latter are Markov population processes with continuous time and discrete state space, and the former take the form of ordinary differential equations.

The propagation of epidemic diseases on scale-free networks has been studied by Pastor-Satorras and Vespignani (2001), who proved the absence of an epidemic threshold and its associated critical behavior. This is the case when there is a large heterogeneity in the degree of contacts of the individuals, as in the case of sexually transmitted diseases.

The analogy of financial contagion with epidemiological models is well evidenced by attention-capturing titles as "When Belgium sneezes, the world catches a cold", "Why Does The U.S. Sneeze When Europe Gets A Cold?", 2 that echo the sentence of Metternich "When France sneezes, Europe catches a cold". 3 Such sentence was pointing out the relevance of France. Nowadays, such studies point out that the interlinking between national economies is so high that even small countries may trigger a crisis. ${ }^{4}$

The difficulty of a straightforward connection between the structure of the economic network and the propagation of shocks, attacks and crises, resides in the fact that they are weighted fully connected networks (complete graphs). Therefore, the crux of the issue is keeping only the most relevant links. Networks examined in Garas et al. (2010) are the Corporate Ownership Network (CON) and the International Trade Network (ITN). Such networks are nearly fully connected, so thresholds are considered for the detection of non trivial structure (Fagiolo et al. 2007). To identify the uneven roles of different countries in the global economic network, the k-shell decomposition method is used.

\footnotetext{
${ }^{1}$ http://phys.org/news/2010-11-belgium-world-cold.html.

${ }^{2}$ http://www.npr.org/2011/08/18/139714695/why-does-the-u-s-sneeze-when-europe-gets-a-cold. ${ }^{3}$ http://www.enotes.com/homework-help/metternich-said-when-france-sneezes-europe-catches120443.

4"The Worlds Most Contagious Countries" https://twistedeconotwist.wordpress.com/2010/11/27/ the-worlds-most-contagious-countries-heres-the-list/.
} 
In graph theory, the concept of $k$-shell is related to the colouring number of a graph. It has been applied in many areas, including social networks (Li et al. 2013). By definition, the $k$-shell of a graph $G$ is a maximal connected subgraph of $G$ in which all vertices have degree at least $k$. Equivalently, it is one of the connected components of the subgraph of $\mathrm{G}$ formed by repeatedly deleting all vertices of degree less than $k$. The $k$-core is the $k$-shell corresponding to the highest $k$. A nucleus of twelve countries has been found to be quite dangerous in the spread of economic crises. Only six of them are large economies, while the other are medium or small economies. Therefore, the position in the network is quite relevant: while it is clear that large economies and big financial institutions affect the entire network, it turns out that the position in the network makes the potential failure of some small-medium economies and institutions could be as dangerous as the failure of the big ones. Such remarks are in line with the paper "Too interconnected to fail" (Markose et al. 2012), that can be grouped in the same discussion line as the papers "Too central to fail" (Battiston et al. 2012), and "Too big to fail" (White 2014). All such papers consider financial networks. The specific characteristics of Credit Default Swaps are examined in Markose et al. (2012). In Battiston et al. (2012) several perspectives contribute to introduce the DebtRank, a centrality measure that states the level of risk of financial institutions. In White (2014) it is shown that the potential loss incurred by saving financial institutions is lower than that of allowing them to fail, due to the nonlinear cascade effects on the network.

Usually, the spread of the economic crisis through a global economic network is examined through the construction of specific models. In Garas et al. (2010) a Susceptible-Infected-Recovered epidemic model is built: the probability of infection depends on the strength of economic relations between the pair of countries, and its strength on the target country. This allows to introduce a ranking of countries in accord to their crises spreading power, that can be used as a further centrality measure. In Chen and Cheng (2015) the credit-debt relation among each couple of financial institutions is specifically modeled.

An interesting phenomenon is the fact that as the average number of connections of each node increases, the probability of default first increases and then drops (Chen and Ghate 2011; Elliott et al. 2014). In fact, the set up of financial links connects the network initially, allowing shocks to propagate; but, as the number of links increases further, organizations are better shielded against other's failures. Beside the specific models for the banks, financial ties among companies are well modeled through the cross-shareholding network. In this context, the integration of a company is the percentage of its shares sold to other companies. It plays a role different from the number of connections: increased dependence on other organizations lowers the sensitivity to own investments (Elliott et al. 2014).

\footnotetext{
${ }^{5}$ Such ideas and concepts became popular through the book A.R. Sorkin (2009) "Too Big to Fail: Inside the Battle to Save Wall Street", Viking Press; and through the movie "Too big to fail" (2011) HBO movie.
} 


\subsection{Synchronization in Networks and Financial Contagions}

The theoretical approach to phase synchronization that was used by Huygens in the seventeenth century (Bennett et al. 2002) was only a first step for the study of complete synchronization of coupled chaotic systems (Wu 2007). In this form of synchronization, the state variable of individuals converge towards each other's value. In the last decades, the research of coupled systems started with Fujisaka and Yamada (1983) and was followed by the research on the synchronization on coupled chaotic systems (Pecora and Carroll 2015). Such research lines were applied to the modelization of many natural phenomena. Synchronization has been shown to have potentially catastrophic effects in constructions (for instance: bridge resonance), earthquakes, economics and finance (Arenas et al. 2008). By way of example, the synchronization of many market agents on short position lead to an increase of supply not well balanced by demand, so triggering price drawdowns and, eventually, large financial crashes (Kaizoji 2009; Malevergne and Sornette 2001; Sieczka et al. 2011; Sornette and Andersen 2002). In behavioral finance, herding is addressed as the main cause of synchronization. In fact, herding behavior shows aggregation and imitation. When it concerns financial market investors, it may lead to the same position either buying, thus causing high rises in prices, or selling, thus causing drawdowns and even crashes in the case of Harmon et al. (2011). However, the reaction on buy or sell are far from being symmetric. Another behavioral finance concept, the so called blindness to small changes, describes the under-reaction to positive or small news, and the over-reaction to negative or quite alarming news. The coupling of this concept with earthquake models has been used for describing the interaction among worldwide markets, and for showing the propagation of market value cascades and avalanches (Andersen et al. 2011; Bellenzier et al. 2016).

The theme of avalanches has been widely studied also in the Self-Organized Criticality (SOC) models. A key example is the Bak and Sneppen (BS) model. The model was developed for describing the co-evolution of species. in that context the disappearance of the less fit species also causes changes in the fitness value of the species that are directly connected to it. The model shows a critical threshold for avalanches. The distribution of avalanches follows a power-law distribution. This model can be seen as a first approximation to the interaction among market agents for the replication of stylized facts (Rotundo and Ausloos 2007; Petroni et al. 2007). While the original BS model was developed on lattices, several extensions have been studied using methods from nonequilibrium statistical mechanics (Lee and Lee 2009; Moreno and Vazquez 2002; Ausloos and Petroni 2014; Rotundo and Scozzari 2009). As expected, the time span and number of network nodes involved in each avalanche depend on the structure of the network. In the framework of systemic risk and financial fragility, it is straightforward to move from the concept of less fit species to the idea of less fit firm, and to generalize from SOC to a general framework for models of cascade and contagion processes on networks (Lorenz et al. 2009; Rotundo and Scozzari 2009). 


\subsection{Games on Networks}

A paradigmatic model in game theory is the Prisoner's Dilemma (PD): two prisoners (players of the game) have to decide wether to remain silent (cooperate) or betray the other player. Each of them incurs in the worst situation when he cooperates and the other betrays. Nash equilibria appear when each player is maximizing their payoff, conditioned to the decision of the other. Therefore, the information available to each player is of utmost relevance. When the 2-player game is extended to $n$ players, it is reasonable to assume that each player has access to some-yet limited-amount of information on the decisions of the others. Therefore, the topology of the network becomes relevant due to the possibility of each player to establish links and access to the information of the other nodes. Statistical mechanics methods are suitable for detecting the optimal Nash equilibria in random as well as scale free networks (Hauert and Szabó 2005; Szabó and Fath 2007).

Of course, many extensions of the PD model have been studied. For instance, evolutionary game theory is designed to capture the essentials of the characteristic interactions among individuals through the evolution of cooperation. The interplay among network structure, clustering and behavioral strategies constitutes an intriguing link between only apparently unrelated disciplines. They are all represented in the framework of game theory, which links the individual behavior to the collective state of equilibrium. On large networks, typical questions are the detection of the percentage of cooperators versus betrayers. Depending on the parameters of the specific model under examination, critical phase transitions may be found. Models have been developed that fall into the universality class of directed percolation on square lattices and mean-field-type transitions on regular small world networks and random regular graphs (Hauert and Szabó 2005).

Evolutionary game theory is the extension of game theory to evolving populations. The population dynamics may concern any evolving set of individual units, ranging from lifeforms in biology to economic or financial agents in economic and financial models. Evolutionary games are not limited to a sequence of game at each step of the evolution, but also consider the frequency of the changes in the strategy (Perc and Szolnoki 2010; Szabó and Fath 2007).

Lattices serve as structure for defining the connection of the players, and open the way to spatial models. In Guan et al. (2006), Szabó and Tőke (1998), and Szabó et al. (2005) the update of a strategy is based on a random selection of nearest neighbours, and the target is understanding the density of cooperators versus betrayers.

Other network structures have been examined. For instance, in Chen et al. (2007), the PD is explored on community networks. Simulation results show that the average degree plays a universal role in cooperation occurring on random networks, smallworld networks, star networks, and scale-free networks (Chen et al. 2007; Du et al. 2009b,c; Pinheiro et al. 2013; Rong et al. 2007; Shang and Wang 2015; Szolnoki et al. 2008; Tang et al. 2006; Wu 2007; Wu et al. 2007). When PD are studied on growing networks of contacts generated via preferential attachment, the correlation among individual increases, and this improves the amount of cooperating 
players (Santos and Pacheco 2005), while the opposite result occurs when a network becomes assortative mixing by degree (Rong et al. 2007). Nash equilibria have been used also for the optimal design of networks, in relation to the optimal transport and optimal navigation problem (Gulyás et al. 2014). Learning has been shown to be another relevant factor for the emergence of cooperators ( $\mathrm{Du}$ et al. 2009c,a). In conclusion, (evolutionary) game theory constitutes a still growing field of application for complex networks, statistical mechanics, and optimization.

\subsection{Clustering of Network Nodes and Geographical Information}

Real life networks-like transport networks and Internet-are quite naturally embedded in a spatial dimension (Gastner and Newman 2006). The information on the geographical location of economic and financial networks has been considered in studies on the Gross Domestic Product (GDP) (Ausloos and Lambiotte 2007; Gligor and Ausloos 2008a,b; Miśkiewicz and Ausloos 2008; Miśkiewicz and Ausloos 2008; Redelico et al. 2009). A technique that is used for both economic and financial data consists in building a network of distances based on the correlation matrix calculated from the time series of the objects under observation. Of course, each of the entries of the empirical estimate of the correlation matrix is non zero, and thus it corresponds to a full network. Thus, techniques for extracting the most relevant information are applied. One of them is the Minimum Spanning Tree (MST). The MST is an acyclic network that connects all the nodes of the networks through the most relevant links. It has been applied on both economic and financial data. For instance, in Miśkiewicz and Ausloos (2008) it is shown that the distance among countries decreases as the year progresses, and this is interpreted as a sign of globalization (Miśkiewicz and Ausloos 2010).

In finance, MST has been used for detecting the backbone of the correlation among financial markets, together with other techniques, like the Maximally Planar Graph and Maximally Filtered Graph (Massara and Matteo 2011; Tumminello et al. 2005, 2007). The combination of tools from statistical physics and network theory allow to test the centrality of economic sector and their evolution during the years (Aste et al. 2010; Pozzi et al. 2008).

Another approach for passing from a complete network to a (usually) complex network consists in keeping only nodes with a connectivity higher than $k$. This leads to $k$-shells and $k$-cores and has been applied to both economic and financial data (Garas et al. 2012). On economic data, it leads to cluster of countries. Another possibility for working on a non complete network is keeping the links that have weights over a specific threshold (Garas et al. 2010).

A different way to explore the economic links among companies, even those belonging to different countries, has been studied in Braha et al. (2011), where a complex networks perspective on interfirm organizational networks is proposed 
by mapping, analyzing and modeling the spatial structure of a large inter-firm competition network across a variety of sectors and industries within the United States. A probabilistic model for the growth of the network is proposed, and it is shown that it is able to reproduce experimentally observed characteristics.

However, the analysis of ownership and control allows to detect economic superentities that span over different countries. In fact, trans-national corporations form a giant bow-tie structure and a large portion of control flows to a small tightly-knit core of financial institutions. This raises new important issues both for researchers and policy makers (Vitali et al. 2011a).

Difficulties in the analysis arise from the fact that such networks show strongly connected components, which lead to circular relations in clusters (Schweitzer et al. 2009 b). The proper value for the ownership through intermediaries can be disentangled through the calculus of integrated ownership (Rotundo and D'Arcangelis 2010b, 2013; Rotundo 2011; Vitali et al. 2011b). Questions on the maximal concentration in markets give rise to nonlinear optimization problems, that also involve the network structure (Rotundo and D'Arcangelis 2013). Networks of companies can be explored also through the board interlocks (D'Errico et al. 2008; Rotundo and D'Arcangelis 2010a). The coupling of information available from such different networks gives an example of application of networks of networks (Dorogovtsev and Mendes 2003).

\section{Conclusions}

In the present contribution we have summarized the main measures of complex networks with an eye on the combination of these topological objects with nonlinear models as a way to describe the phenomenology of complex systems. Specifically, we have analysed epidemiological compartmental models in combination with complex networks and we have briefly reviewed the main applications these formalisms have found in financial contagions. Moreover, we reviewed on games on networks and also on the usefulness of network nodes for analyzing geographical aspects of economic and financial networks.

Acknowledgements This work has been performed in the framework of COST Action IS1104 "The EU in the new economic complex geography: models, tools and policy evaluation". L.M.V. thanks the financial support of the Dirección Xeral de Saude Pública of the Xunta de Galicia (Spain) and the many fruitful discussions — and personal friendship — of X. Hervada and A. Malvar. L.M.V. wants to explicitly and strongly acknowledge the many contributions and useful discussions with his very close friends and colleagues J. Carrete and C. Ricoy-Riego, without whom this work would surely have not been done and life would be totally different. 


\section{References}

Albert R, Barabási AL (2002) Statistical mechanics of complex networks. Rev Mod Phys 74:47-97

Albert R, Jeong H, Barabasi A (2000) Error and attack tolerance of complex networks. Nature 406(6794):378-382

Almeida-Neto M, Guimarães P, Guimarães Jr PR, Loyola RD, Ulrich W (2008) A consistent metric for nestedness analysis in ecological systems: reconciling concept and measurement. Oikos 117:1227-1239

Andersen JV, Nowak A, Rotundo G, Parrott L, Martinez S (2011) "price-quakes" shaking the world's stock exchanges. PloS One 6(11):e26472

Araujo AIL, Corso G, Almeida AM, Lewinsohn TM (2010) An analytic approach to the measurement of nestedness in bipartite networks. Physica A 389:1405-1411

Arenas A, Díaz-Guilera A, Kurths J, Moreno Y, Zhou C (2008) Synchronization in complex networks. Phys Rep 469(3):93-153

Aste T, Shaw W, Matteo TD (2010) Correlation structure and dynamics in volatile markets. New J Phys 12(8):085009

Atmar W, Patterson B (1993) The measure of order and disorder in the distribution of species in fragmented habitat. Oecologia 96(3):373-382

Ausloos M, Lambiotte R (2007) Clusters or networks of economies? A macroeconomy study through gross domestic product. Phys A Stat Mech Appl 382(1):16-21

Ausloos M, Petroni F (2014) Threshold model for triggered avalanches on networks. Preprint. arXiv: 14014270

Barabási AL, Albert R (1999) Emergence of scaling in random networks. Science 286:509-512

Barrat A, Barthlemy M, Vespignani A (2008) Dynamical processes on complex networks, 1st edn. Cambridge University Press, New York

Barthélemy M, Barrat A, Pastor-Satorras R, Vespignani A (2005) Dynamical patterns of epidemic outbreaks in complex heterogeneous networks. J Theor Biol 235(2):275-288

Battiston S, Puliga M, Kaushik R, Tasca P, Caldarelli G (2012) DebtRank: too central to fail? Financial networks, the FED and systemic risk. Sci Rep 2:541

Bellenzier L, Vitting AJ, Rotundo G (2016) The world's stock exchange seen as network of coupled oscillators. In: Economic modelling (in press)

Bennett M, Schatz MF, Rockwood H, Wiesenfeld K (2002) Huygens's clocks. Proc R Soc Lond A Math Phys Eng Sci 458(2019):563-579

Boccaletti S, Latora V, Moreno Y, Chavez M, Hwang DU (2006) Complex networks: structure and dynamics. Phys Rep 424:175-308

Braha D, Stacey B, Bar-Yam Y (2011) Corporate competition: a self-organized network. Soc Networks 33(3):219-230

Chen PY, Cheng SM (2015) Sequential defense against random and intentional attacks in complex networks. Phys Rev E 91:022805

Chen X, Ghate A (2011) Financial contagion on power law networks. http://dx.doi.org/10.2139/ ssrn. 1751143

Chen X, Fu F, Wang L (2007) Prisoner's dilemma on community networks. Phys A Stat Mech Appl 378(2):512-518

Chua L (1998) CNN: a paradigm for complexity. World Scientific series on nonlinear science: monographs and treatises. World Scientific, Singapore. https://books.google.es/books?id= G86zMVD3yNYC

Costa LDF, Oliveira ON Jr, Travieso G, Rodrigues FA, Villas Boas PR, Antiqueira L, Correa Rocha LE (2011) Analyzing and modeling real-world phenomena with complex networks: a survey of applications. Adv Phys 60(3):329-412

D’Errico M, Grassi R, Stefani S, Torriero A (2008) Shareholding networks and centrality: an application to the Italian financial market. In: Naimzada A, Stefani S, Torriero A (eds) Network, topology and dynamics. Theory and applications to economics and social systems. Springer, Berlin, pp 215-228 
Dorogovtsev SN, Mendes JFF (2003) Evolution of networks: from biological nets to the internet and WWW (Physics). Oxford University Press, Inc., New York

$\mathrm{Du} \mathrm{WB}, \mathrm{Cao} \mathrm{XB}, \mathrm{Hu} \mathrm{MB}$ (2009a) The effect of asymmetric payoff mechanism on evolutionary networked prisoner's dilemma game. Phys A Stat Mech Appl 388(24):5005-5012

Du WB, Cao XB, Hu MB, Yang HX, Zhou H (2009b) Effects of expectation and noise on evolutionary games. Phys A Stat Mech Appl 388(11):2215-2220

Du WB, Cao XB, Zhao L, Hu MB (2009c) Evolutionary games on scale-free networks with a preferential selection mechanism. Phys A Stat Mech Appl 388(20):4509-4514

Elliott M, Golub B, Jackson MO (2014) Financial networks and contagion. Am Econ Rev 104(10):3115-53

Erdös P, Rényi A (1959) On random graphs I. Publ Math 6:290-297

Fagiolo G, Reyes J, Schiavo S (2007) On the topological properties of the world trade web: a weighted network analysis. Papers, arXiv.org. http://EconPapers.repec.org/RePEc:arx:papers: 0708.4359

Fujisaka H, Yamada T (1983) Stability theory of synchronized motion in coupled-oscillator systems. Prog Theor Phys 69(1):32-47

Garas A, Argyrakis P, Rozenblat C, Tomassini M, Havlin S (2010) Worldwide spreading of economic crisis. New J Phys 12:113043

Garas A, Schweitzer F, Havlin S (2012) A $k$-shell decomposition method for weighted networks. New J Phys 14:083030

Gastner MT, Newman ME (2006) The spatial structure of networks. Eur Phys J B-Condensed Matter Complex Syst 49(2):247-252

Gligor M, Ausloos M (2008a) Clusters in weighted macroeconomic networks: the eu case. introducing the overlapping index of gdp/capita fluctuation correlations. Eur Phys J B 63(4):533-539

Gligor M, Ausloos M (2008b) Convergence and cluster structures in eu area according to fluctuations in macroeconomic indices. Papers, arXiv.org. http://EconPapers.repec.org/RePEc: arx:papers:0805.3071

Guan JY, Wu ZX, Huang ZG, Xu XJ, Wang YH (2006) Promotion of cooperation induced by nonlinear attractive effect in spatial prisoner's dilemma game. Europhys Lett 76(6):1214

Gulyás A, Bíró J, Körösi A, Rétvári G, Krioukov D (2014) Complex networks as Nash equilibria of navigation games. Preprint arXiv:1412.7229

Harmon D, de Aguiar MA, Chinellato DD, Braha DD, Epstein I, Bar-Yam Y (2011) Predicting economic market crises using measures of collective panic. Preprint. http://ssrn.com/abstract= 1829224 or http://dx.doi.org/10.2139/ssrn.1829224

Hauert C, Szabó G (2005) Game theory and physics. Am J Phys 73(5):405-414

Holme P (2005) Core-periphery organization of complex networks. Phys Rev E 72:046111

Kaizoji SDT (2009) Chap Market Bubbles and Crashes

Kermack WO, McKendrick AG (1927) A contribution to the mathematical theory of epidemics. Proc R Soc Lond A Math Phys Eng Sci 115(772):700-721

Lee KE, Lee JW (2009) Avalanches of Bak-Sneppen coevolution model on directed scale-free network. Fractals 17(02):233-237

Li J, Cao L, Wang C, Tan KC, Liu B, Pei J, Tseng VS (2013) Trends and applications in knowledge discovery and data mining: PAKDD 2013 Workshops DMApps, DANTH, QIMIE, BDM, CDA, CloudSD, Golden Coast, QLD. Springer Publishing Company, Incorporated

López-Pintado D (2008) Diffusion in complex social networks. Game Econ Behav 62:573-590

Lorenz J, Battiston S, Schweitzer F (2009) Systemic risk in a unifying framework for cascading processes on networks. Eur Phys J B 71(4):441-460

Malevergne Y, Sornette D (2001) Multi-dimensional rational bubbles and fat tails. Quant Financ 1(5):533-541

Markose S, Giansante S, Shaghaghi AR (2012) "Too interconnected to fail” financial network of \{US\} \{CDS \} market: topological fragility and systemic risk. J Econ Behav Organ 83(3):627646 
Massara GP, Di Matteo T (2011) Network filtering for big data: triangulated maximally filtered graph. Preprint. arXiv: 150502445

Milgram S (1967) The small-world problem. Psychol Today 1:60-67

Miśkiewicz J, Ausloos M (2008) Correlation measure to detect time series distances, whence economy globalization. Phys A Stat Mech Appl 387(26):6584-6594

Miśkiewicz J, Ausloos M (2008) Correlation measure to detect time series distances, whence economy globalization. Phys A Stat Mech Appl 387(26):6584-6594

Miśkiewicz J, Ausloos M (2010) Has the world economy reached its globalization limit? Phys A Stat Mech Appl 389(4):797-806

Moreno Y, Vazquez A (2002) The Bak-Sneppen model on scale-free networks. Europhys Lett 57(5):765

Newman M (2003) The structure and function of complex networks. SIAM Rev 45:167-256

Newman M, Watts D, Barabási AL (2006) The structure and dynamics of networks. Princeton University Press, Princeton

Nåsell I (2002) Stochastic models of some endemic infections. Math Biosci 179(1):1-19

Pastor-Satorras R, Vespignani A (2001) Epidemic spreading in scale-free networks. Phys Rev Lett 86:3200-3203

Pastor-Satorras R, Vespignani A (2002) Epidemic dynamics in finite size scale-free networks. Phys Rev E 65:035,108

Pastor-Satorras R, Rubi M, Díaz-Guilera A (eds) (2003) Statistical mechanics of complex networks. Springer, Berlin

Pecora LM, Carroll TL (2015) Synchronization of chaotic systems. Chaos 25(9):097611

Perc M, Szolnoki A (2010) Coevolutionary games-a mini review. Biosystems 99(2):109-125

Petroni F, Ausloos M, Rotundo G (2007) Generating synthetic time series from Bak-Sneppen coevolution model mixtures. Phys A Stat Mech Appl 384(2):359-367

Pinheiro FL, Vasconcelos V, Santos F, Pacheco JM (2013) Self-organized game dynamics in complex networks. ECAL - General Track 61-62

Pozzi F, Aste T, Rotundo G, Di Matteo T (2007) Dynamical correlations in financial systems. In: Microelectronics, MEMS, and Nanotechnology. International Society for Optics and Photonics, p 68021E

Redelico FO, Proto AN, Ausloos M (2009) Hierarchical structures in the gross domestic product per capita fluctuation in Latin American countries. Phys A Stat Mech Appl 388(17):3527-3535

Rong Z, Li X, Wang X (2007) Roles of mixing patterns in cooperation on a scale-free networked game. Phys Rev E 76:027101

Rotundo G (2011) Centrality measures in shareholding networks. In: Use of risk analysis in computer-aided persuasion. NATO science for peace and security series, vol 88 . IOS Press, Amsterdam, pp 12-28

Rotundo G, Ausloos M (2007) Microeconomic co-evolution model for financial technical analysis signals. Phys A Stat Mech Appl 373:569-585

Rotundo G, D'Arcangelis AM (2010a) Network analysis of ownership and control structure in the Italian stock market. Adv Appl Stat Sci 2:255-273

Rotundo G, D'Arcangelis AM (2010b) Ownership and control in shareholding networks. J Econ Interaction Coord 5:191-219

Rotundo G, D'Arcangelis AM (2014) Network of companies: an analysis of market concentration in the Italian stock market. Quality Quantity 48(4):1893-1910

Rotundo G, Scozzari A (2009) Co-evolutive models for firms dynamics. In: Networks, topology and dynamics, vol 613. Springer, Berlin, pp 143-158

Santos FC, Pacheco JM (2005) Scale-free networks provide a unifying framework for the emergence of cooperation. Phys Rev Lett 95(9):098104

Schweitzer F, Fagiolo G, Sornette D, Vega-Redondo F, Vespignani A, White DR (2009a) Economic networks: the new challenges. Science 325(5939):422

Schweitzer F, Fagiolo G, Sornette D, Vega-Redondo F, White DR (2009b) Economic networks: what do we know and what do we need to know? Adv Complex Syst 12:407-422 
Serrano MA, Krioukov D, Boguñá M (2008) Self-similarity of complex networks and hidden metric spaces. Phys Rev Lett 100:078701

Shang L, Wang XF (2007) Evolutionary minority game on complex networks. Phys A Stat Mech Appl 377(2):616-624

Sieczka P, Sornette D, Holyst JA (2011) The Lehman brothers effect and bankruptcy cascades. Eur Phys J B 82(3-4):257-269

Sornette D, Andersen JV (2002) A nonlinear super-exponential rational model of speculative financial bubbles. Int J Mod Phys C 13(02):171-187

Szabó G, Fath G (2007) Evolutionary games on graphs. Phys Rep 446(4):97-216

Szabó G, Tóke C (1998) Evolutionary prisoner's dilemma game on a square lattice. Phys Rev E 58(1):69

Szabó G, Vukov J, Szolnoki A (2005) Phase diagrams for an evolutionary prisoner's dilemma game on two-dimensional lattices. Phys Rev E 72(4):047107

Szolnoki A, Perc M, Danku Z (2008) Towards effective payoffs in the prisoner's dilemma game on scale-free networks. Phys A Stat Mech Appl 387(8):2075-2082

Tang CL, Wang WX, Wu X, Wang BH (2006) Effects of average degree on cooperation in networked evolutionary game. Eur Phys J B-Condensed Matter Complex Syst 53(3):411-415

Tumminello M, Aste T, Di Matteo T, Mantegna RN (2005) A tool for filtering information in complex systems. Proc Natl Acad Sci USA 102(30):10421-10426

Tumminello M, Di Matteo T, Aste T, Mantegna R (2007) Correlation based networks of equity returns sampled at different time horizons. Eur Phys J B 55(2):209-217

Vitali S, Glattfelder JB, Battiston S (2011a) The network of global corporate control. PLoS One 6:e25995

Vitali S, Glattfelder JB, Battiston S (2011b) The network of global corporate control. PloS One 6(10):e25995

Wang XF (2002) Complex networks: topology, dynamics and synchronization. Int J Bifurcation Chaos 12(05):885-916

Watts D, Strogatz S (1998) Collective dynamics of small-world networks. Nature 393:440-442

White LJ (2014) The basics of'too big to fail'. NYU Working Paper No. 2451/33564. http://papers.ssrn.com/sol3/Papers.cfm?abstract_id=2405433

Wu CW (2007) Synchronization in complex networks of nonlinear dynamical systems, vol 76. World Scientific, Singapore

Wu ZX, Guan JY, Xu XJ, Wang YH (2007) Evolutionary prisoner's dilemma game on BarabásiAlbert scale-free networks. Phys A Stat Mech Appl 379(2):672-680 